\title{
Deficiencia de folatos y su asociación con defectos de cierre del tubo neural en el norte de México
}

\author{
Martha Rodríguez-Morán, M.C., M. en C., Jesús Fernando Guerrero-Romero, M.C., ${ }^{(1)}$ \\ Martín Parra-Q uezada, M.C., ${ }^{(2)}$ María de Jesús Segura-Pineda, M.C., ${ }^{(3)}$ \\ Margarita Levario-C arrillo, M.C., ${ }^{(4)}$ Elma Ivonne Sotelo-Ham, M.C. ${ }^{(5)}$
}

\section{Rodríguez-Morán M, Guerrero-Romero JF, Parra-Quezada M, Segura-Pineda MJ, Levario-Carrillo M, Sotelo-Ham El. Deficiencia de folatos y su asociación con defectos de cierre del tubo neural en el norte de México.}

Salud Publica Mex 1998;40:474-480.

\begin{abstract}
Resumen
Objetivo.Valorar la asociación de la deficiencia de folatos y otros factores de riesgo con la ocurrencia de defectos de cierre del tubo neural (DCTN), en la población rural del norte de México (Chihuahua, Durango y Zacatecas). Material y métodos. Se hizo un estudio multicéntrico de casos y controles. Se consideraron como casos a los recién nacidos vivos (RN V) y a los muertos con DCTN, y como controles, a los RNV sanos, no malformados. Se determinó la exposición a factores de riesgo conocidos, estableciendo su asociación con los DCTN, con un modelo de análisis múltiple de regresión logística. Resultados. LOS factores de riesgo asociados a DCTN fueron: la deficiencia de folatos (RM 11.1; IC 95\% 1.2-106.2, $p=0.04$ ); el antecedente, en embarazos previos, de productos con DCTN (RM 3.3; IC 95\% 1.1-18.8, $p=0.05$ ), y óbitos (RM 7.1; IC 95\% $1.1-46.3, p=0.04)$. Conclusiones La deficiencia de folatos constituye uno de los principales factores de riesgo asociado a los DCTN en la población rural del norte de México. Es necesario llevar a cabo más investigaciones para determinar la contribución de otros factores de riesgo y establecer las medidas preventivas adecuadas.
\end{abstract}

Palabras clave: defectos del tubo neural; ácido fólico;México

\author{
Rodríguez-Morán M, Guerrero-Romero JF, \\ Parra-Quezada M, Segura-Pineda MJ, \\ Levario-Carrillo M, Sotelo-Ham El. \\ Folic acid deficiency and its relationship \\ with neural tube defects \\ in northern Mexico.
}

Salud Publica Mex 1998;40:474-480.

\begin{abstract}
A bstract
Objective To evaluate folic acid deficiency and other risk factors and their relationship with the occurrence of neural tube defects (NTD), in the rural population of northern Mexico (C hihuahua, D urango and Zacatecas). Material and methods. A multicentric case-control study was performed. Cases were both live and stillborn with NTD, and controls were healthy newborns without congenital malformations. Exposure to known risk factors was determined, establishing its association with N TD using multiple logistic regression analysis. Results. Risk factors associated to N TD were: folic acid deficiency (O R 11.1; CI 95\% 1.2-106.2, $p=0.04)$; the antecedents of previous N TD pregnancies (OR 3.3; $\mathrm{Cl} 95 \%$ 1.1-18.8, $\mathrm{p}=0.05$ ) and stillbirths (OR 7.1; $\mathrm{Cl}$ $95 \% 1.1-46.3, p=0.04)$. Conclusions Folic acid deficiency is one of the major risk factors associated to N TD among the rural population of northern Mexico. Fur ther investigations are necessary to determine the role of involved risk factors and implement adequate preventive measures.
\end{abstract}

Key words: neural tube defects; folic acid; Mexico

(1) Investigador asociado C. Hospital General de Zona N o.1, Instituto Mexicano del Seguro Social (IMSS), Delegación D urango, México.

(2) Hospital General de Sub-Zona N 0.16, IMSS, Delegación Chihuahua, México.

(3) Hospital General N 0. 15, IMSS, Delegación Chihuahua, México.

(4) Coordinación Delegacional de Investigación, IMSS, D elegación Chihuahua, México.

(5) Coordinación Delegacional de Investigación, IMSS, D elegación Zacatecas, México.

Fecha de recibido: 2 de septiembre de 1997 - Fecha de aprobado: 10 de agosto de 1998 Solicitud de sobretiros: Dr. Jesús Fernando Guerrero Romero. Siqueiros 225 esq. Castañeda, 34000 zona centro, Durango, D go., México. Correo electrónico: guerrero@ omanet.com.mx 
as malformaciones congénitas (MC) son un problema de salud pública ${ }^{1}$ que repercute en el núcleo familiar y en la sociedad..$^{2}$ La causa de la mayoría de las malformaciones es multifactorial, ${ }^{3,4}$ lo cual implica la interacción de factores genéticos y ambientales; sin embargo, se estima que aproximadamente $69 \%$ de las malformaciones congénitas tienen una etiología desconocida. $^{3}$

Alrededor de uno de cada 50 recién nacidos vivos (RNV) y de uno de cada nueve recién nacidos muertos (RNM) presentan una o más malformaciones, algunas de tipo letal, entre las que destaca la anencefalia. ${ }^{5}$

Los defectos de cierre del tubo neural (DCTN) se desarrollan durante la tercera y la cuarta semanas posconcepcionales, ${ }^{6}$ lo que da lugar a malformaciones con diversos grados de severidad y pronóstico; en la mayoría de los casos, estas últimas conducen a la invalidez o a la muerte en los primeros días de la vida. ${ }^{6,7}$ Los DCTN más frecuentes son la espina bífida, la anencefalia y el mielomeningocele, ${ }^{8,9}$ en cuya etiología se han detectado deficiencias nutricionales, déficit de ácido fólico, antecedentes maternos de diabetes y de infertilidad, alteraciones genéticas y, por último, exposición a fármacos. ${ }^{7}$

La frecuencia de malformaciones congénitas registra variaciones significativas en los diferentes estudios que al respecto se han hecho; ${ }^{6}$ esas variaciones se deben, en general, a los aspectos metodológicos de definición y detección de los defectos congénitos. ${ }^{10}$ En abortos espontáneos, la incidencia de DCTN varía de $0.8 \times 1000$-en la serie reportada por Canún-Serrano y colaboradores- ${ }^{11}$ a 2.13 x 100 -en la de Arredondo-de Arreola y colaboradores-.$^{12}$ Por otro lado, en relación con los RNV, Mutchinick y colaboradores ${ }^{5}$ notifican una incidencia de anencefalia de $0.39 \times 1000$, mientras que Jiménez-Balderas y colaboradores ${ }^{2}$ indican una prevalencia de DCTN de 1.51 x 1000 RNV, y Walss-Rodríguez y colaboradores, ${ }^{7}$ una de 5.2 x 1000 RNV. En Durango, en una serie que incluyó RNV y RNM, la incidencia de DCTN notificada por Rodríguez-Morán y colaboradores ${ }^{13}$ fue de $13.2 \times 1000 \mathrm{RN}$ al año. En las diferentes series analizadas, entre los DCTN destaca la mayor frecuencia de anencefalia ${ }^{5,10-13}$ y mielomeningocele. ${ }^{13}$

El objetivo de este trabajo fue valorar la asociación entre la deficiencia de folatos y la ocurrencia de DCTN en la población rural de los estados de Chihuahua, Durango y Zacatecas.

\section{Material y métodos}

Se desarrolló un estudio multicéntrico de casos y controles, en el que se incluyeron habitantes del área rural de los estados de Chihuahua, Durango y Zacatecas,
México, que acudieran durante el periodo de enero de 1995 a diciembre de 1996 a atención del parto a los hospitales rurales Nos. 18, 36 y 82 del sistema IMSS-Solidaridad, y al Hospital General de Zona No. 16 del Instituto Mexicano del Seguro Social en la ciudad de Cuauhtémoc, Chihuahua. Se seleccionaron estas instituciones para participar en el estudio, porque se consideró que ofrecen atención médica a población abierta del área rural del norte de México, y que cuentan con personal médico especializado en los departamentos de pediatría y gineco-obstetricia.

Se consideraron como casos los RNV y RNM que presentaron DCTN, y como controles, los RNV sanos, no malformados, de los dos partos que siguieron a aquel en el que se registró la malformación. Por cada caso se consideraron dos controles.

Para estandarizar los criterios de diagnóstico y clasificación de los DCTN y de los procedimientos del estudio, antes de iniciarlo se celebraron reuniones con los médicos encargados de examinar a los recién nacidos (RN). Con el propósito de uniformar, en la medida de lo posible, la información recabada, sólo se consideraron como DCTN la anencefalia y el mielomeningocele. El coordinador del estudio en la ciudad de Durango fue el encargado de concentrar la información para capturarla, analizarla e interpretarla.

En cada unidad participante, un médico cegado a la condición de caso-control aplicó un cuestionario a las madres de los productos de ambos grupos, en el periodo inmediato de posparto y previo consentimiento informado; ello se hizo con la finalidad de determinar la presencia de los factores de riesgo asociados a los DCTN, considerando como tales: la edad materna $<18$ años y >35 años; la presencia de enfermedades crónicas o agudas durante el embarazo; la administración de medicamentos como anticonvulsivantes, hipoglucemiantes orales, etcétera; la exposición a insecticidas agrícolas; el antecedente de abortos; óbitos o productos con DCTN en embarazos previos, y la deficiencia de folatos, que se estableció con el análisis de frotis de sangre periférica, tomados en el momento del trabajo de parto, y que un médico hematólogo y otro internista revisaron de manera independiente y cegada. Se consideró que había deficiencia de folatos cuando en los frotis se podía observar una hipersegmentación del núcleo de los neutrófilos ${ }^{14,15} \mathrm{y}$, al menos, dos de las siguientes alteraciones: anisocitosis y poiquilocitosis, macroovalocitos y deformación de las plaquetas, ${ }^{14}$ en las que la estimación de su presencia es de carácter subjetivo.

Por medio de entrevistas directas y de la revisión de los expedientes se recabó información sobre la ingesta de suplementos vitamínicos durante el em- 
barazo. Además, se preguntó acerca del lugar de procedencia, sobre historia de tabaquismo, consumo de alcohol u otras drogas, y antecedentes obstétricos.

El tamaño de la muestra se estimó con base en la formula ${ }^{16}$

$$
n=(Z \alpha / 2+Z \beta)^{2} \mathrm{P}(1-\mathrm{P})(\mathrm{r}+1) / \delta^{2} \mathrm{r} \text { donde }
$$

$\mathrm{P}=$ el valor ponderado de $p^{2}$ y $p^{1}\left[\left(\mathrm{P}=p^{2}+\mathrm{r} p^{1}\right) /(1+\mathrm{r})\right]$

$\mathrm{r}=$ la razón entre el número de controles por caso

$p^{2}=$ la proporción de los casos expuestos a los factores de riesgo

$p^{1}=$ la proporción de los controles expuestos a los factores de riesgo

$\delta=$ el valor nulo de las diferencias entre las proporciones.

De esta forma se requieren 53 casos y 106 controles, según los resultados de una muestra piloto constituida por los casos y controles integrados en el primer año del estudio, donde se captaron $35 \mathrm{RN}$ con DCTN y 70 RNV sanos; en cuatro casos y un control se establecieron, mediante los frotis sanguíneos de las madres, cambios morfológicos compatibles con deficiencia de folatos $\left(p^{2}=4 / 35\right.$ y $\left.p^{1}=1 / 70\right)$, considerando un valor de $\alpha=0.05, \beta=0.20$ y $\delta=0.10$, y una razón controles / $\operatorname{casos}(\mathrm{r}=2)$.

\section{A nálisis estadístico}

Las diferencias entre los grupos se estimaron con el cálculo de $t$ de Student (o U Mann Whitney) para las variables recabadas en escala numérica en muestras independientes y con la prueba exacta de Fisher para las variables nominales.

La variación interobservador en la interpretación de los frotis de sangre periférica se determinó con la prueba de Kappa. ${ }^{17}$ En el análisis se incluyeron únicamente los casos en que hubo acuerdo interobservador.

La fuerza de asociación entre los factores de riesgo y los DCTN se estableció con el cálculo de la razón de momios (RM), a partir de un modelo múltiple de regresión logística, en el que se incluyeron como variables independientes: la edad de la madre $(<18$ y $>35$ años), considerando como grupo de referencia al de 19 a 35 años de edad; la ingesta de medicamentos (que se consideró como positiva en los casos en que se documentó la administración de anticonvulsivantes, hipoglucemiantes orales, antibióticos, betabloqueadores y dipirona durante el primer trimestre del embarazo); el antecedente de óbitos, abortos y productos con DCTN en embarazos previos; la exposición a productos agro- químicos, y la deficiencia de folatos. Todas estas variables se consideraron como dicotómicas, en las que el grupo de comparación fue el que no tenía la exposición al factor de riesgo. En todos los casos se consideró un intervalo de confianza de $95 \%$.

\section{Resultados}

Durante el periodo de estudio se identificaron 62 casos de RN con DCTN y 124 RNV en el grupo control. No hubo rechazos de participar en el estudio; sin embargo, en seis niños con DCTN y en uno del grupo control, la toma del frotis de sangre periférica no se pudo analizar por extendido deficiente, o bien, los datos recolectados fueron incompletos, por lo que no se incluyeron en el análisis; por tanto, se integraron $56 \mathrm{RN}$ con DCTN y $123 \mathrm{RNV}$ sanos, para una proporción casos: controles de 1:2.2.

De los 56 casos, 39 (69.6\%) correspondieron a anencefalia, $12(21.4 \%)$ a mielomeningocele y cinco $(8.9 \%)$ a anencefalia con mielomeningocele. De los $44 \mathrm{RN}$ con anencefalia, $28(63.6 \%)$ fueron productos muertos al nacimiento y $16(36.4 \%)$ nacieron vivos, pero todos fallecieron en las 48 horas posteriores al parto; de los $\mathrm{RN}$ con mielomeningocele, dos $(16.6 \%)$ nacieron muertos y $10(83.4 \%)$ estaban vivos a las 72 horas del nacimiento.

Las madres de los productos con DCTN eran más jóvenes que las del grupo control (22.7 \pm 5.0 años vs $26.6 \pm 6.5$ años, $p=0.04$ ), tenían menor tasa de paridad $(2.7 \pm 1.9$ gestas vs $3.1 \pm 2.5$ gestas, $p=0.58)$ y mayor frecuencia de abortos $(1.1 \pm 0.2$ vs $0.5 \pm 0.2, p=0.05)$ y óbitos ( $1.4 \pm 0.5$ vs $0.15 \pm 0.9, p=0.05)$.

Se identificó hábito de tabaquismo y consumo de alcohol en seis $(10.7 \%)$ de las madres de los casos y en cinco $(4.0 \%)$ de los controles $(p=0.10)$. Hubo una mayor frecuencia de grupo sanguíneo $\mathrm{A}+$ en las madres de los niños con DCTN (10.7 vs 3.2\%, $p=0.07$ ). Las madres de los productos con DCTN presentaron un porcentaje más elevado de enfermedades crónicas (diabetes tipo II o hipertensión arterial sistémica) o agudas (infecciones de vías urinarias o respiratorias), motivo por el cual recibieron tratamiento médico durante el embarazo (cuadro I).

En las madres de ambos grupos no se registraron casos de desnutrición ni se documentó antecedente de suplementación con vitaminas o ácido fólico durante el primer trimestre del embarazo. Se identificaron alteraciones morfológicas en el frotis sanguíneo compatibles con deficiencia de folatos en ocho $(14.3 \%)$ de las madres de RN con DCTN y en una $(0.8 \%)$ del grupo control $(\mathrm{RM}=20.3)$, en las que hubo acuerdo interobservador; por otro lado, en un caso se presentó 
desacuerdo entre los observadores, por lo que para efectos del análisis no se incluyó en el estudio. El valor de Kappa fue de 0.940. Tanto el antecedente de productos con DCTN, como el de óbitos en embarazos previos, fueron más frecuentes en el grupo de casos. Por otro lado, en ninguno de los grupos se identificó el antecedente de tratamiento con anticonvulsivantes (difenil-hidantoína, carbamazepina, ácido valproico, etc.). Los demás factores de riesgo estudiados no mostraron asociación con la ocurrencia de DCTN (cuadro I).

El análisis múltiple de regresión logística mostró una asociación significativa de los DCTN con la deficiencia de folatos y el antecedente de óbitos y productos con DCTN en embarazos previos (cuadro II).

\section{Discusión}

En comparación con los informes sobre países étnicamente semejantes al nuestro, la prevalencia de anencefalia en México es significativamente más alta, ${ }^{10}$ por lo que es necesario incrementar los conocimientos sobre los aspectos genéticos y agentes teratogénicos ambientales asociados a estos defectos. ${ }^{4}$
La población blanco de este estudio la constituyeron habitantes del área rural, en zonas calificadas como de marginación social ${ }^{18}$ del norte del país (Chihuahua, Durango y Zacatecas) donde, de acuerdo con estudios previos, ${ }^{7,13}$ se ha detectado una elevada prevalencia de recién nacidos con DCTN. Así entonces, una limitación de este estudio es que sólo se incluyó en el mismo a núcleos poblacionales en condiciones específicas de vida, de las que se han señalado como relacionadas a DCTN la clase social baja ${ }_{1}^{19}$ la dificultad de acceso a los centros de atención médica, las deficiencias nutricionales y de suplementación vitamínica durante el primer trimestre del embarazo, y la baja escolaridad de los padres. ${ }^{8,9,20}$

Una desventaja de los estudios multicéntricos es la diversidad de criterios, experiencia y conocimientos del personal que participa, lo que determina que los estudios puedan tener un alto grado de variabilidad ${ }^{2}$ que obliga a realizar esfuerzos para disminuir las potenciales fuentes de dicha variabilidad. En ese contexto, dentro de este trabajo se consideró como DCTN sólo a los casos de anencefalia y mielomeningocele cuyo reconocimiento clínico no ofrece dificultades diagnósticas.

\section{Cuadro I}

ANÁlisis biVARIAdo de los faCtORES de RIESGo ASOCIADOS CON LA OCURRENCIA DE DEFECTOS de Cierre del tubo neural. Norte de México, 1995-1996

\begin{tabular}{|c|c|c|c|c|c|}
\hline & $\begin{array}{l}\text { Casos } \\
\text { n (\%) }\end{array}$ & $\begin{array}{c}\text { Controles } \\
n(\%)\end{array}$ & $\begin{array}{l}\text { Razón de } \\
\text { momios* }\end{array}$ & IC $95 \%$ & $p$ \\
\hline Edad $<18$ y $>35$ años & $13(23.2)$ & $23(18.6)$ & 1.3 & $0.6-3.0$ & 0.62 \\
\hline \multicolumn{6}{|l|}{ Antecedentes obstétricos } \\
\hline Abortos & $5(8.9)$ & $8(6.5)$ & 1.4 & $0.4-5.1$ & 0.76 \\
\hline O bitos & $10(17.9)$ & $7(5.7)$ & 3.6 & $1.7-11.3$ & 0.02 \\
\hline $\mathrm{DCTN}^{\dagger}$ & $7(12.5)$ & $2(1.6)$ & 8.6 & $1.6-34.0$ & 0.004 \\
\hline D eficiencia de folatos & $8(14.3)$ & $1(0.8)$ & 20.3 & $2.5-95.6$ & 0.0004 \\
\hline Tabaquismo & $6(10.7)$ & $5(4.0)$ & 2.8 & $0.7-11.3$ & 0.10 \\
\hline Consumo de alcohol & $6(10.7)$ & $5(4.0)$ & 2.8 & $0.7-11.3$ & 0.10 \\
\hline Exposición a agroquímicos & $3(5.3)$ & $2(1.6)$ & 3.4 & $0.4-19.2$ & 0.17 \\
\hline \multicolumn{6}{|l|}{ Enfermedades en el embarazo } \\
\hline Crónicas & $4(7.1)$ & $5(4.1)$ & 1.8 & $0.4-8.2$ & 0.46 \\
\hline Agudas & $6(10.7)$ & $8(6.5)$ & 1.7 & $0.5-5.8$ & 0.37 \\
\hline \multicolumn{6}{|l|}{ Medicamentos usados ${ }^{\ddagger}$} \\
\hline Ampicilina & $2(3.6)$ & $3(2.4)$ & 1.5 & $0.3-11.3$ & 0.65 \\
\hline Trimetoprim-sulfametoxasol & $3(5.3)$ & $7(5.7)$ & 0.9 & $0.2-4.0$ & 1.0 \\
\hline Dipirona & $2(3.6)$ & $4(3.2)$ & 1.1 & $0.2-7.3$ & 1.0 \\
\hline Acido acetilsalicílico & $5(8.9)$ & $8(6.5)$ & 1.4 & $0.4-5.0$ & 0.54 \\
\hline Betabloqueadores & $2(3.6)$ & $3(2.4)$ & 1.5 & $0.3-11.3$ & 0.65 \\
\hline Alfametildopa & $2(3.6)$ & $2(1.6)$ & 2.2 & $0.4-15.6$ & 0.59 \\
\hline Insulina & $2(3.6)$ & $1(0.8)$ & 4.5 & $0.5-41.7$ & 0.23 \\
\hline
\end{tabular}




\section{Cuadro II \\ ANÁLISIS DE REgRESIÓN LOGÍSTICA MÚLTIPLE}

\begin{tabular}{|c|c|c|c|}
\hline ctores de riesgo & Razón de momios & IC $95 \%$ & $\mathrm{p}$ \\
\hline Edad $<18$ años* & 0.9 & $0.8-1.0$ & 0.12 \\
\hline Edad >35 años* & 1.2 & $0.9-2.9$ & 0.08 \\
\hline Ingesta de medicamentos ${ }^{\ddagger}$ & 0.7 & $0.1-2.7$ & 0.21 \\
\hline Abortos ${ }^{\ddagger \S}$ & 5.1 & $0.6-43.5$ & 0.07 \\
\hline$\overline{0 \text { bitos }}{ }^{\ddagger \S}$ & 7.1 & $1.1-46.3$ & 0.04 \\
\hline D efectos de cierre del tubo neural $\left.\right|^{\ddagger \S}$ & 3.3 & $1.1-18.8$ & 0.05 \\
\hline Exposición a agroquímicos ${ }^{\ddagger}$ & 1.1 & $0.9-19.6$ & 0.17 \\
\hline Deficiencia de folatos ${ }^{\ddagger}$ & 11.1 & $1.2-106.2$ & 0.04 \\
\hline \multicolumn{4}{|c|}{$\begin{array}{l}\text { * Considerando como grupo de comparación al de } 19 \text { a } 35 \text { años de edad } \\
\text { ₹ Considerando como grupo de comparación a las madres sin exposición } \\
\text { al factor de riesgo } \\
\text { § Documentados en embarazos previos }\end{array}$} \\
\hline
\end{tabular}

En estudios previos se ha demostrado la importancia de la asociación entre DCTN y la deficiencia de folatos en el embarazo, ${ }^{6,19}$ durante el cual las fuentes de folatos pueden ser el aporte dietético, así como la suplementación vitamínica. Respecto al primero, en este trabajo no se midió el contenido de la dieta habitual; en lo tocante a la suplementación vitamínica, se ha descrito la posibilidad de que exista un sesgo de memoria acerca de la ingesta de suplementos vitamínicos durante el embarazo ${ }^{21}$ y de que la madre se refiera a ese antecedente como el responsable del factor de la malformación. El hecho de recordar una exposición a factores de riesgo ocurrida meses antes del evento que se estudia puede estar influido por el intervalo de tiempo entre la exposición y el momento de recabar la información; ${ }^{22}$ sin embargo, la posibilidad de que haya ocurrido este sesgo de memoria en la población que se estudia se reduce en virtud de que la única fuente de atención médica que se tiene en esa área rural es la que ofrece el sistema IMSS-Solidaridad, de tal manera que se pudo verificar la prescripción de suplementos durante el periodo de gestación.

Por otro lado, aun cuando es preferible la medición directa de los niveles de folatos en sangre para determinar su disminución, ésta se puede estimar de manera confiable a partir de cambios detectables en el frotis de sangre periférica, en el que la hipersegmentación neutrofílica refleja el balance negativo de folatos, ${ }^{14,15}$ y es un hallazgo tan característico que una sola célula con un núcleo de seis o más lóbulos constituye una sospecha clínica de anemia megaloblástica. ${ }^{14}$ En este estudio la estimación de la deficiencia de folatos se realizó al final del embarazo y no durante la tercera y la cuarta semanas del mismo, cuando ocurren los tras- tornos del desarrollo que culminan con DCTN en el producto; ${ }^{6}$ esta circunstancia constituye una limitación del estudio, ya que la exposición que se midió no correspondió al periodo de riesgo. Al respecto es necesario tener en cuenta que en la embarazada la deficiencia del ácido fólico se presenta por la elevada demanda del feto en desarrollo, que tal deficiencia tarda meses en generarse y que la anemia se establece en forma crónica; el proceso para que ésta desaparezca amerita un tratamiento sustitutivo ${ }^{14}$ de uno a dos meses de duración, por lo que se puede asumir que las mujeres en las que se detectó deficiencia de folatos cursaban con ésta en forma crónica considerando, además, que en ningún caso se documentó el antecedente de terapia sustitutiva de folatos. Por otro lado, éticamente no se puede incluir en estudios de investigación a mujeres con deficiencia de folatos documentada en forma previa al embarazo o durante las primeras semanas de gestación, por el alto riesgo que tienen de desarrollar productos con DCTN, ${ }^{23}$ de manera que esta limitación de temporalidad a que se hace referencia se ha presentado en otros estudios. ${ }^{24,25}$

La asociación entre deficiencia de ácido fólico y DCTN es controversial; por un lado, se ha descrito una asociación directa ${ }^{19,20,22} \mathrm{y}$, por el otro, algunos autores, ${ }^{21,26,27}$ aduciendo que existen problemas de interpretación derivados del sesgo de memoria, señalan que sería difícil establecer si tal asociación depende de los suplementos multivitamínicos por sí mismos, o bien, de otros factores nutricionales o de estilos de vida asociados con ellos. ${ }^{9}$ En un ensayo clínico aleatorio realizado recientemente con mujeres que tenían antecedente de productos con DCTN, se demostró que la administración de $4 \mathrm{mg}$ de ácido fólico al día, durante las seis semanas previas a la concepción y a lo largo del primer trimestre del embarazo, disminuye en $72 \%$ la recurrencia de DCTN. ${ }^{28}$

Puesto que la administración de suplementos de folatos durante el periodo periconcepcional puede disminuir la recurrencia y ocurrencia de DCTN, su uso es altamente recomendable..$^{29-31}$ Por otra parte, tomando en cuenta la confluencia de factores sociales y económicos adversos a la población blanco bajo estudio, que dificultan su acceso a las unidades de atención médica, es igualmente recomendable la orientación dietética para favorecer un aporte adecuado de folatos mediante el consumo de alimentos que lo contengan (como el hígado, las espinacas, la lechuga romana, las legumbres, el germen de trigo, los granos integrales, los cacahuates y la levadura), ${ }^{6,10,32}$ sobre todo en el periodo periconcepcional y el primer trimestre del embarazo, lo que podría disminuir la incidencia de este problema en la población rural de México. 
La asociación de los DCTN con el antecedente de óbitos y productos con DCTN en embarazos previos, que se documentó en este estudio, pudiera relacionarse con la presencia de alteraciones genéticas; sin embargo, por las limitaciones técnicas para realizar las mediciones adecuadas, no se estableció la participación de este factor de riesgo en la recurrencia y ocurrencia de los DCTN que se observó. Además, cabe suponer que algunos factores que favorecen la aparición de DCTN, como los hábitos dietéticos de la madre gestante ${ }^{2}$-no determinados en este trabajo- y el sesgo de memoria relacionado con variables como la exposición a agroquímicos, la ingesta de medicamentos durante el embarazo, etcétera, influyen de manera determinante en la génesis del problema que se estudia, lo que plantea la necesidad de realizar estudios específicos para medir la participación de estos y otros factores en la ocurrencia de los DCTN.

Finalmente, si bien el tamaño de la muestra se estableció con base en criterios derivados de cálculos matemáticos específicos, ${ }^{16}$ habrá que tener en cuenta que únicamente se identificó deficiencia de folatos en ocho de las madres del grupo de casos y en una del grupo control, por lo que, a pesar de todo, existe la posibilidad de que se hayan cometido errores en el análisis, derivados de una muestra insuficiente. Por ende, es necesario llevar a cabo estudios que incluyan una muestra mayor y con mediciones más específicas, para determinar la contribución de los diferentes factores relacionados con este problema y evaluar el establecimiento de las medidas de prevención adecuadas.

\section{Agradecimientos}

Se agradece el apoyo que brindaron para la realización de este estudio los doctores Jesús Alvaro Huízar Marín, jefe del Departamento de Pediatría del Hospital General de Zona No.1 del IMSS en Zacatecas; Angel Ledezma Nieves, director del Hospital Rural de IMSS-Solidaridad en Vicente Guerrero, Durango, y Francisco Sandoval Herrera, coordinador del Programa IMSS-Solidaridad en Durango.

\section{Referencias}

1. Arredondo-de Arreola $G$, Rodríguez-Bonito $R$, Treviño-Alanís MG, Arreola-Arredondo B, Astudillo-C astillo G, Russildi JM. Malformaciones congénitas en recién nacidos vivos. Bol Med Hosp Infant Mex 1990;47: 822-827.

2. Jiménez-Balderas E, Salamanca-Gómez F, Martínez-A pac S, Bracho-Solís M. Estudio de malformaciones congénitas en 105825 nacimientos consecutivos. Bol Med Hosp Infant Mex 1985;42:744-748.
3. Hernández-Arriaga JL, C ortés-Gallo G, Aldana-Valenzuela C, RamírezHuertaAC . Incidencia de malformaciones congénitas externas en el Hospital de Ginecopediatría N 0.48 en León, G uanajuato. Bol Med Hosp Infant Mex 1991;48:717-721.

4. Cedeño-Rincón R, León A, Romero R. Epidemiología de las malformaciones congénitas externas en una maternidad de Venezuela. Bol Med Hosp Infant Mex 1996;53:117-122.

5. Mutchinick 0, Lisker R, Babinski V. Programa mexicano de "Registro y vigilancia epidemiológica de malformaciones congénitas externas". Salud Publica Mex 1988;30:88-100.

6. García-Morales M, Limón-Luque LM, Barrón-Vallejo J, González-Morales $M E, K$ ably-Ambe A. U so periconcepcional de ácido fólico en la prevención de defectos del tubo neural: conceptos actuales. Ginecol 0 bstet Mex 1996;64:418-421.

7.W alss-Rodríguez R, Reyes-G arnica A, A costa-Calderón AR, Murra-Remusat JE, Rodríguez-Rivera E. Epidemiología de los defectos congénitos del tubo neural en la ciudad de Torreón, Coahuila. Rev Med Inst Mex Seguro Soc 1990;28:265-268.

8.W iswell TE, Tuttle D, N ortham R, Simonds G. Major congenital neurologic malformations. A 17-year survey. Am J D is Child 1990;144:61-67.

9. Yen $Y$, Khoury $M$, Erickson D, James L,W aters $G$, Berry R. The changing epidemiology of neural tube defects. United States, 1968-1989. Am J D is Child 1992;146:857-861.

10. Mutchinick 0 . Epidemiología de las malformaciones congénitas. $\mathrm{Gac}$ Med Mex 1996;131:152-160.

11. C anún-Serrano S, Saavedra-O ntiveros D, C havira-Estefan S,A ndradeTapia F. Malformaciones congénitas en diferentes etapas del desarrollo intrauterino. Ginecol O bstet Mex 1990;58:1-4.

12. Arredondo-de Arreola G, López-Serna N ,Treviño-Alanís MG, Russildi JM, Arreola-Arredondo B, Borrego S. Frecuencia de malformaciones congénitas externas en abortos. Bol Med Hosp Infant Mex 1992;49:32-38.

13. Rodríguez-Morán M, Guerrero-Romero JF, Sandoval-Herrera F, Ledezma-N ieves A. Prevalencia de malformaciones congénitas en una población socioeconómica baja. Rev Invest Med SS, IMSS 1996;1:16-23.

14. Babior BM, Bunn F. A nemias megaloblásticas. En: Isselbacher KJ, Braunwald E,W ilson JD, Martin JB, Fauci A, Kasper D, ed. Principios de medicina interna. 13a. edición. Madrid: Interamericana McG raw-Hill, 1994; vol. Il:1986-1993.

15. Pietrzik KF, Thorand B. Folate economy in pregnancy. $\mathrm{N}$ utrition 1997;13:975-977.

16. Mejía-Aranguré JM, Fajardo-Gutiérrez A, Gómez-D elgado A, CuevasU rióstegui ML, Hernández-Hernández DM, Garduño-Espinoza J et al. El tamaño de muestra: un enfoque práctico en la investigación clínica pediátrica. Bol Med Hosp Infant Mex 1995;52:381-391.

17. Loría A. Estadística mínima. VI. El manejo de datos no paramétricos. Lab-Acta 1990;21:15-18.

18. Flores-Alvarado A. Diagnóstico de salud en las zonas marginadas rurales de México, 1986-1991. México, D.F.: Instituto Mexicano del Seguro Social, 1992:107-109.

19. Milunsky A, Jick H, Jick S, Bruell C, MacLaughlin D, Rothman $K$ et al. Multivitamin/folic acid supplementation in early pregnancy reduces the prevalence of neural tube defects. JAMA 1989;262:2847-2852.

20. W illett W. Folic acid and neural tube defect: $C$ an't we come to closure? Am J Public Health 1992;82:666-668.

21. Mills JL, Rhoads G, Simpson JL, C unningham GC, Conley MR, Lassman $M R$ et al.The absence of a relation between the periconceptional use of vitamins and neural-tube defects. N Engl J Med 1989;321:430-435.

22. Mulinare J, Cordero J, Erickson D, Berry R. Periconceptional use of multivitamins and the occurrence of neural tube defects. JAMA 1988; 260:3141-3145.

23. Daly S, Mills J, Molloy A, Conley M, Lee Y, Kirke PN et al. Minimum effective dose of folic acid for food fortification to prevent neural-tube defects. Lancet 1997;350:1666-1669. 
24. Breown J, Jacobs D, Hartman T, Barosso G, Stang J, Gross M et al. Predictors of red cell folate level in women attempting pregnancy. JAMA 1997;277:545-552.

25. Daly LE, Kirke PN, Molloy A, W eir DG, Scott JM. Folate levels and neural tube defects. JAMA 1995;274:1698-1702.

26. Mills J, Rhoads G, Simpson J, C unningham G.Vitamins during pregnancy and neural tube defects. JAMA 1990;263:2747-2748.

27. Shapiro S, Mitchell A, Werler M. Letter to the editor. JAMA 1990;263: 2748.

28. Medical Research Council Vitamin Study Research Group. Prevention of neural tube defects; results of the Medical Research Council Vitamin Study. Lancet 1991;338:131-137.
29. Eskes TK. Folates and the fetus. Eur J O bstet Gynecol Reprod Biol 1997;71:105-111.

30. Eskes TK, Steegers-Theunissen RP. Primary prevention of neural-tube defects with folic acid. Eur J Obstet Gynecol Reprod Biol 1994;53: 147-152.

31. Eskes TK. Possible basis for primary prevention of birth defects with folic acid. Fetal Diagn Ther 1994;9:149-154.

32. W igertz K, Svensson UK, Jagerstad M. Folate and folate-binding protein content in dairy products. J Dairy Res 1997;64:239-252. 